\title{
Systematic analysis of coronary artery disease datasets revealed the potential biomarker and treatment target
}

\author{
Yan Shi ${ }^{1, *}$, Sijin Yang ${ }^{2, *}$, Man Luo ${ }^{3}$, Wei-Dong Zhang ${ }^{4}$ and Zun-Ping Ke ${ }^{5}$ \\ ${ }^{1}$ Department of Emergency, The Affiliated Huai'an Hospital of Xuzhou Medical University and The Second People's Hospital \\ of Huai'an, Huai'an, China \\ ${ }^{2}$ Department of Heart Encephalopathy, Affiliated Traditional Chinese Medicine Hospital, Southwest Medical University, \\ Luzhou, China \\ ${ }^{3}$ Department of Emergency, Huai'an First People's Hospital, Nanjing Medical University, Huai'an, China \\ ${ }^{4}$ Department of Cardiology, People's Hospital of Xuyi, Jiangsu, Xuyi, China \\ ${ }^{5}$ Department of Cardiology, The Fifth People's Hospital of Shanghai, Fudan University, Shanghai, China \\ * Co-first authors \\ Correspondence to: Wei-Dong Zhang, email: xyxrmyy@163.com \\ Zun-Ping Ke, email: kzprenmin@163.com
}

Keywords: coronary artery disease, systematic analysis, microarray, gene expression omnibus (GEO), neutrophil degranulation Received: February 13, $2017 \quad$ Accepted: March 29, $2017 \quad$ Published: April 26, 2017

Copyright: Shi et al. This is an open-access article distributed under the terms of the Creative Commons Attribution License 3.0 (CC BY 3.0), which permits unrestricted use, distribution, and reproduction in any medium, provided the original author and source are credited.

\section{ABSTRACT}

Coronary artery disease caused about 1 of every 7 deaths in the United States and early prevention was potential to decrease the incidence and mortality. We aimed to figure the genes involving in the coronary artery disease using meta-anlaysis. Five datasets of coronary heart disease from GEO series were retrieved and data preprocessing and quality control were carried out. Moderated t-test was used to decide the differentially expressed genes for a single dataset. And the combined p-value using systematic-analysis methods were conducted using MetaDE. The pathway enrichment was carried out using Reactome database. Protein-protein interactions of the identified differentially expressed genes were also analyzed using STRING v10.0 online tool. After removing unidentified or intermediate samples and a total of 238 cases and $\mathbf{1 8 9}$ matched or partially matched control from five microarray datasets were retrieved from GEO. Six different quality control measures were calculated and PCA biplots were plotted in order to visualize the quantitative measure. The first two PCs captured $91 \%$ of the variance and we decided to include all of the datasets for systematic analysis. Using the FDR cut-off as 0.1 , nine genes, including LFNG, ID3, PLA2G7, FOLR3, PADI4, ARG1, IL1R2, NFIL3 and MGAM, were differentially expressed according to maxP. Their protein-protein interactions showed that they were closely connected and 24 Reactome pathways were related to coronary artery disease. We concluded that pathways related to immune responses, especially neutrophil degranulation, were associated with coronary heart disease.

\section{INTRODUCTION}

Coronary artery disease caused about 1 of every 7 deaths, which corresponded to 375295 deaths, in the United States in 2011 [1]. It was estimated that about 635,000 and 300,000, respectively, Americans have a new or recurrent coronary attach every year. Death rates of coronary heart disease have fallen from 1968 to the present and about $47 \%$ of the decrease in deaths caused by coronary artery disease contributed to treatments, including secondary preventive therapies after myocardial infarction or revascularization, initial treatments for acute 
Table 1: The GEO datasets used in this study

\begin{tabular}{lcccc}
\hline GEO Accession & Platform & Source DOI & control & case \\
\hline gse20681 & GPL4133 & $10.1186 / 1755-8794-5-58$ & 99 & 99 \\
gse20680 & GPL4133 & $10.1186 / 1755-8794-4-26$ & 52 & 87 \\
gse29532 & GPL5175 & $10.1016 /$ j.cca.2013.03.011 & 6 & 8 \\
gse42148 & GPL13607 & NA & 11 & 13 \\
gse48060 & GPL570 & $10.1016 /$ j.yjmcc.2014.04.017 & 21 & 31 \\
\hline
\end{tabular}

Table 2: Quantitative quality control measures of coronary heart disease studies

\begin{tabular}{llccccccc}
\hline Dataset & Study & IQC & EQC & CQCg & CQCp & AQCg & AQCp & Rank \\
\hline 1 & gse20680 & 6.25 & 0.9 & 1.84 & 8.58 & 1.7 & 9.27 & 2.5 \\
2 & gse20681 & 4.5 & 1.1 & 10.91 & 27.02 & 4.29 & 10.78 & 1.5 \\
3 & gse29532 & 2.85 & 0.53 & 0.24 & 2.42 & 0.08 & 0.01 & 4.5 \\
4 & gse42148 & 0.61 & 1.1 & 2.39 & 9.34 & 1.6 & 2.73 & 2.83 \\
5 & gse48060 & 4.91 & 0.91 & 0.24 & 0.84 & 0.8 & 3.73 & 3.67 \\
\hline
\end{tabular}

myocardial infarction or unstable angina and so forth [2]. It suggested that early prevention of coronary heart disease was effective to some extent.

Microarray analysis has been used as a practical approach to study gene expression changes, which may help the early diagnosis of coronary heart disease [3]. Despite their great promise, a lot of studies have reported that findings pf microarray data were not reproducible or were sensitive to the data perturbations [4, 5]. Even worse, microarray used over 10 thousand probes on tens or hundreds of samples, which exacerbated the accuracy of the potential predictors.

As a result, the systematic analysis was used to increase the reliability and generalizability of results. Through systematic analysis, we aimed to obtain a more precise set of differentially expressed genes, and analyzed their biological functions. In this study, we utilized the five public microarray datasets from Gene Expression Omnibus (GEO) repository [6] to figure out the genes which were differentially expressed in patients with coronary heart disease and control using combined $\mathrm{p}$ values and try to give suggestions on the biomarkers for the early prevention and treatment according to the functions of these genes.

\section{RESULTS}

\section{Quality of datasets}

Five microarray datasets of patient samples with coronary heart diseases for which matched clinical information was available were obtained from GEO by using GEOquery (Table 1). After removing unidentified or intermediate samples and a total of 238 cases and 189 matched or partially matched control were selected for further analysis (Figure 1A). The detailed information of these five microarray datasets was summarized in Table 1 and Figure 1B.

Six quality control measures were calculated (Table 2) and PCA biplots (Figure 2A) were plotted in order to visualize the quantitative measure. The first two PCs also captured a high percentage of variance (91\%), and the studies were more scattered in the plot. For example, GSE20680 had better scores in IQC, CQCp and AQCp while GSE20681 had better performance in CQCg, CQCp, AQCg and AQCp. GSE20681 and GSE48060 had relatively poor performance. One concern is that the EQC were poor in all five datasets, which suggested that the genes involved in coronary heart disease might not be finely defined in Biocarta pathways. Considering the PCA biplots, the six quality control criteria and the limited datasets, we decided to include all of the datasets for systematic analysis.

\section{Differentially expressed genes}

Five main systematic analysis methods by combining p-value in MetaDE package were carried out including maxP, minP, roP, AW and Fisher. The counts of differentially expressed genes by each independent datasets and by selected combined p-value were listed in Table 3. Totally seven differentially expressed genes were detected by maxP and roP evaluation criteria, respectively, using detection competency curves and false discovery rate (FDR) cut-off less than 0.05. If the FDR cut-off was set as 0.1, 9 genes were differentially expressed (Figure 2C). 
These nine genes had different patterns across the samples. For example, LFNG and ID3 were highly expressed in control samples, while ARG1 and IL1R2 were highly expressed in patients with coronary heart disease. Such expression patterns matched their functions which were reviewed in detail in the discussion section. The number of differentially expressed genes were plotted as a function of false discovery rate FDR in the analysis of five different datasets and the five different systematic analysis algorithms (Figure 2B). And it showed that GSE48060 and GSE42148 performed the best.

\section{Function analysis}

The pathways shared by at least three datasets were plotted as heatmap (Figure 3 ) with a cutoff of FDR lower than 0.05 using Reactome database [15]. We identified 24 pathways related to coronary artery disease by these criteria. Notably, most of these pathways were associated with the immune system. Neutrophil degranulation seemed to be the most important pathways associated with coronary heart disease.

\section{Protein-protein interactions}

The protein-protein interactions of the identified differentially expressed genes showed that they were closely connected and played a central role in vivo (Figure 4B). For example, ARG1, LFNG, and PLA2G7 are hubs for this network which suggested their important function in human body.
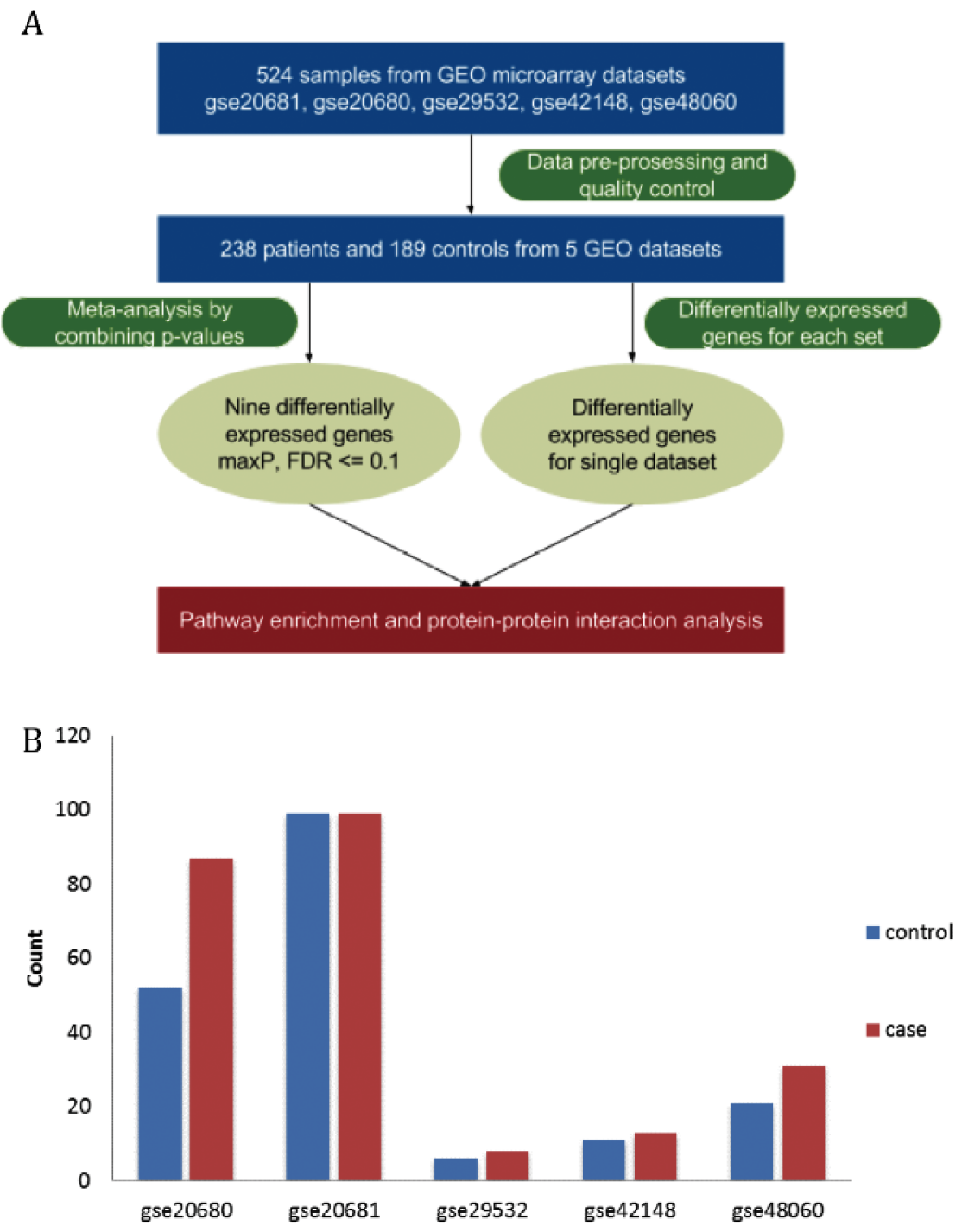

Figure 1: Overview of the systematic analysis and datasets of the coronary heart disease. (A) The workflow of this study. (B) The number of cases (red) and controls (blue) in the five datasets of the coronary heart disease. 

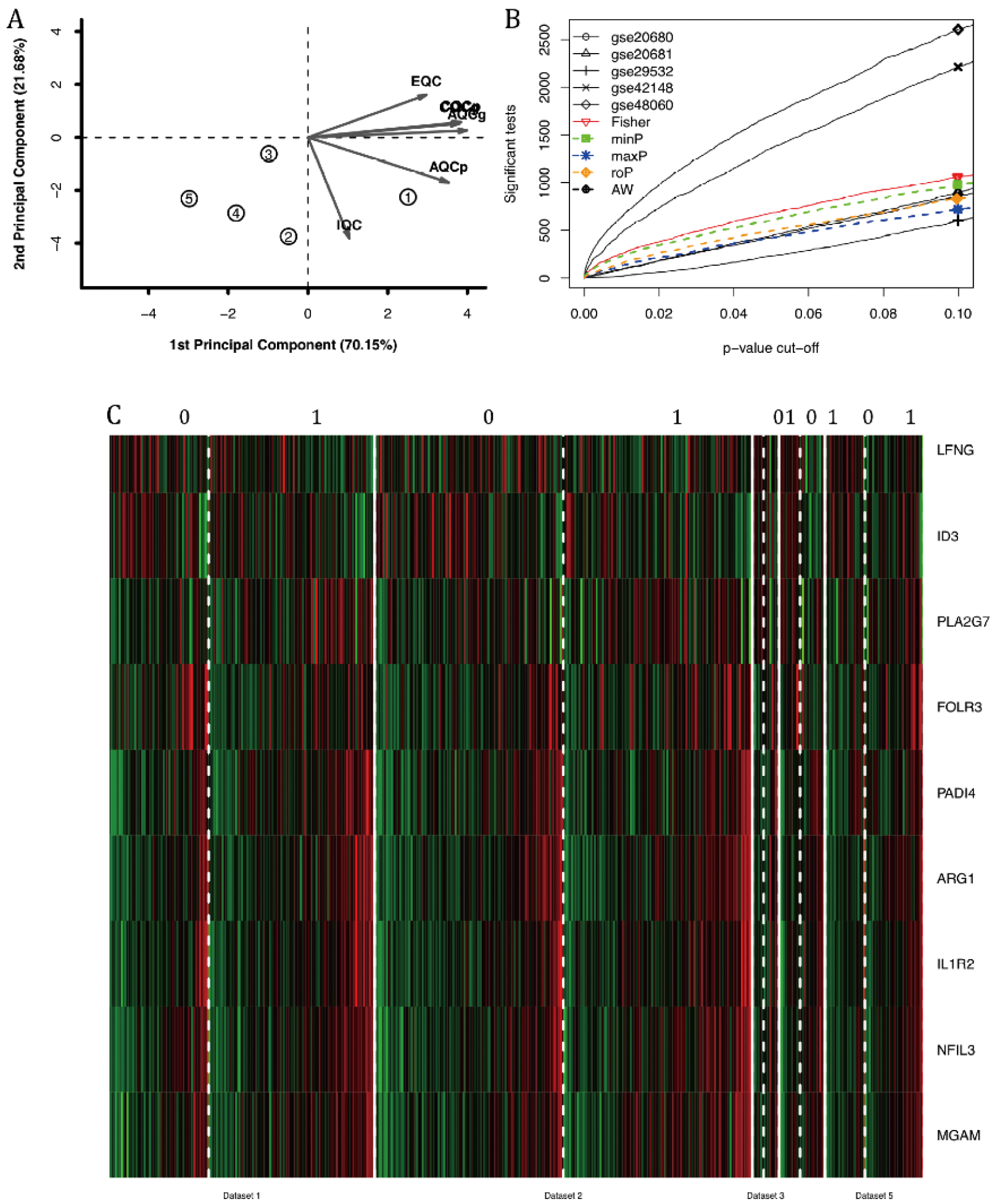

Dåasel 1
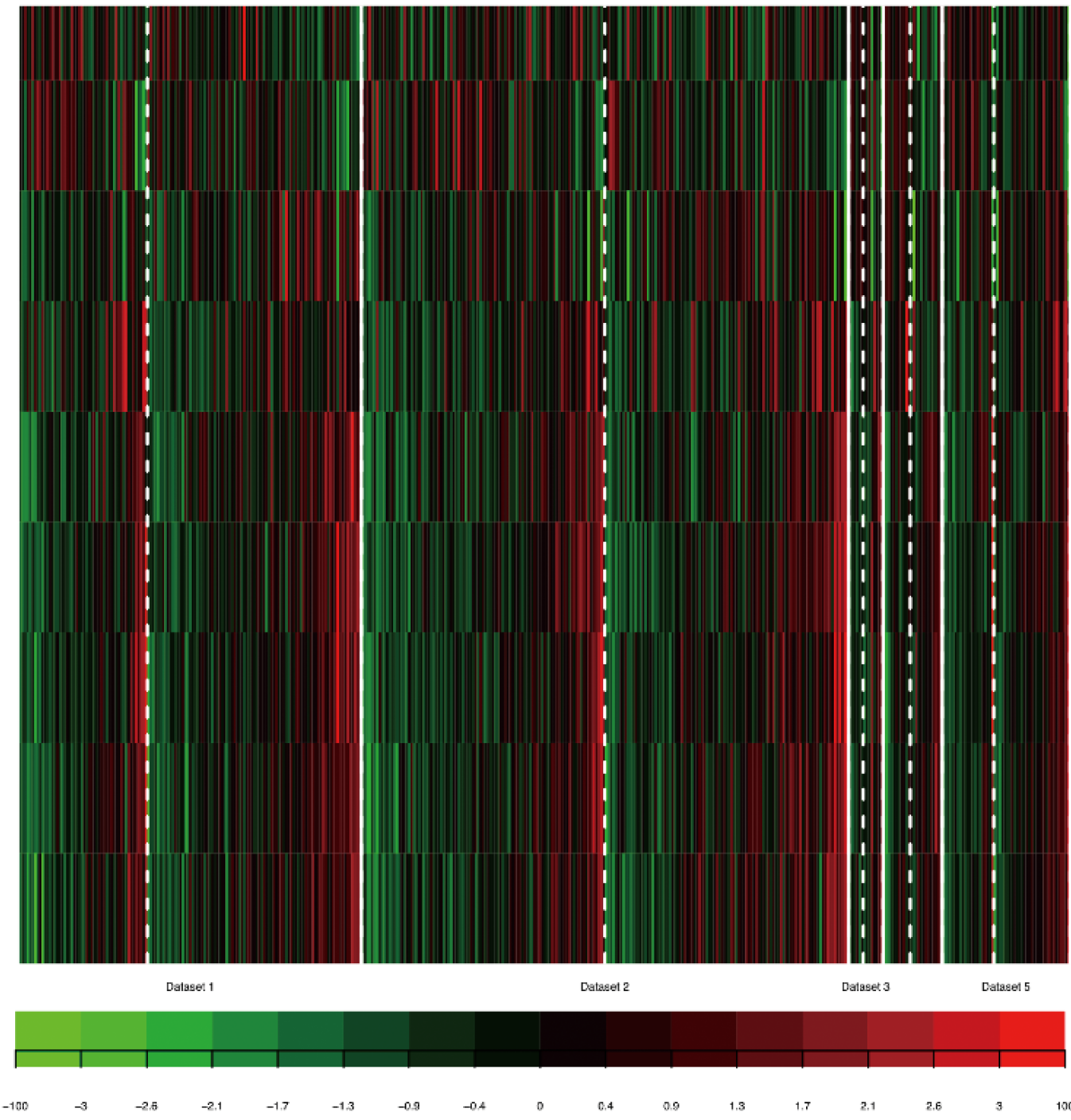

Figure 2: The systematic analysis of differentially expressed genes between patients with coronary heart diseases and controls by combining p-value. (A) PCA biplot of six quality control measures in five datasets. (B) The number of differentially expressed genes plotted as FDR in the analysis of five different datasets. (C) The heatmap identifying the differentially expressed gene in cases and controls subjected to maxP systematic analysis when FDR was lower than 0.1. 
Table 3: The number of differentially expressed genes in the five datasets of coronary heart disease using moderated-t test and meta-analysis combined $p$ value

\begin{tabular}{lccccccc}
\hline Cutoff & gse20681 & gse20680 & gse29532 & gse42148 & gse48060 & roP & maxP \\
\hline $\mathrm{p}<=0.01$ & 70 & 89 & 17 & 506 & 633 & 163 & 136 \\
$\mathrm{p}<=0.05$ & 415 & 418 & 227 & 1445 & 1697 & 500 & 423 \\
$\mathrm{FDR}<=0.01$ & 0 & 0 & 0 & 1 & 3 & 2 & 2 \\
$\mathrm{FDR}<=0.05$ & 0 & 2 & 0 & 9 & 109 & 7 & 7 \\
\hline
\end{tabular}

\section{DISCUSSION}

\section{Differentially expressed genes in patients with coronary heart disease}

With the microarray expression data, we can figure out the genes whose expression levels help diagnose the diseases or identify the most suitable treatment. However, microarray data sometimes were not reproducible or were too sensitive to the mildest data perturbations [3]. Moreover, over ten thousand probes are investigated in only tens or hundreds of biological samples, which increase the false positive targets dramatically. As a result, it is a good idea to utilize different datasets of similar experiment designs to decrease the false positives.

In this study, we combined five datasets of coronary heart disease and used the combined maxP value to figure out nine important and reproducible genes which were differentially expressed between patients and controls. These nine genes were LFNG, ID3, PLA2G7, FOLR3, PADI4, ARG1, IL1R2, NFIL3 and MGAM. These genes were differentially expressed in all five microarray datasets, which suggested that their significant roles in the coronary heart disease. When the protein-protein interactions of these nine genes were investigated, we found that there genes had many interactors (Figure 4B), which indicated that their critical roles in vivo. Notably, LFNG, PLA2G7, and ARG1, which were annotated by the red arrows, were obviously hubs in the protein-protein interaction networks.

LFNG (lunatic fringe) is a member of the fringe gene family and acts in the Notch receptor pathway, which regulated macrophage activation and cardiovascular calcification [7]. Mutations on Notch1 pathways led to
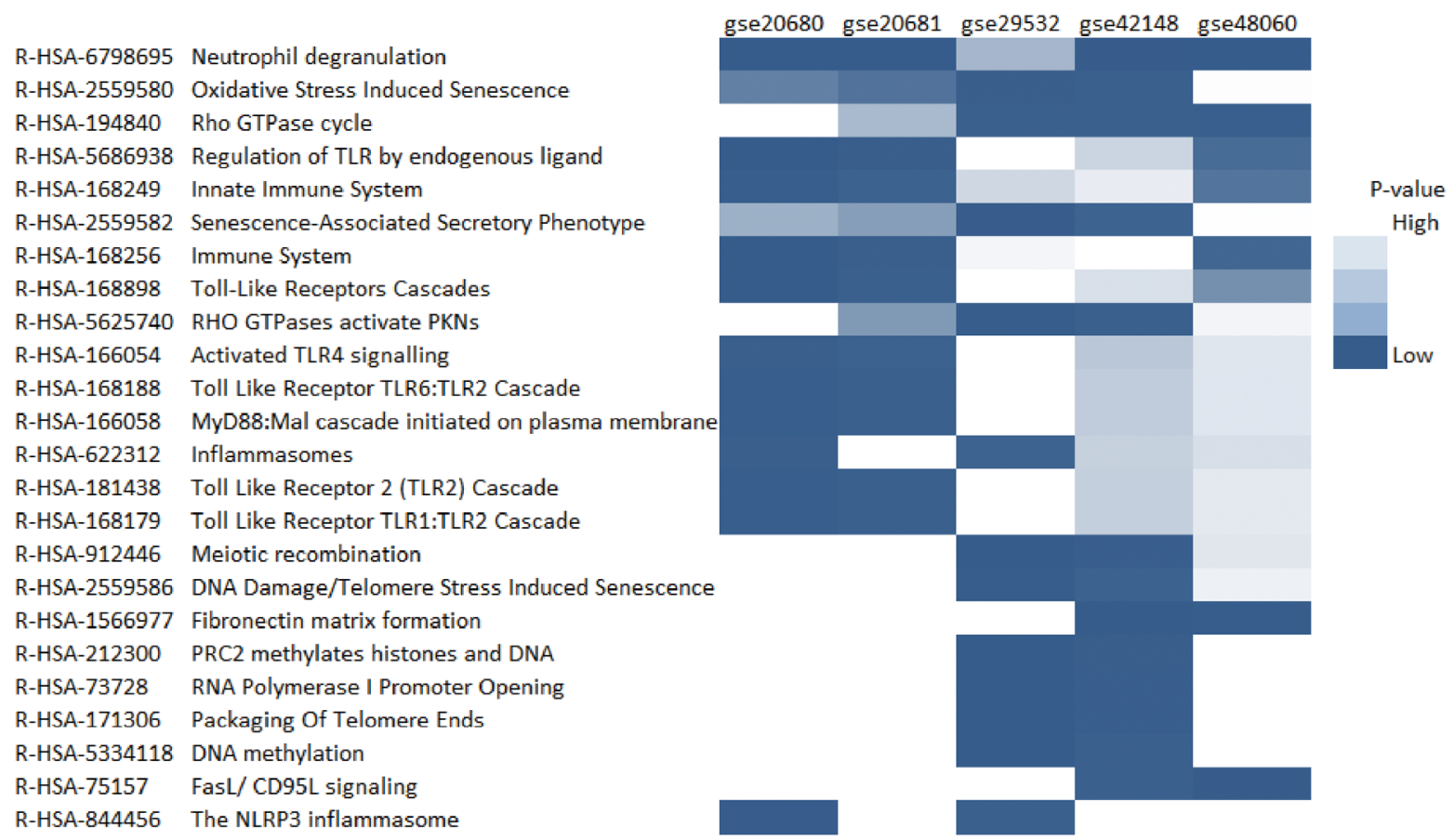

Figure 3: The heatmap of enriched pathways from Reactome. Only the pathways which were significantly enriched with the cutoff of FDR lower than 0.05 in at least two datasets were shown in the plot. 
high rate of coronary heart disorders like stenosis and calcification. ID3 (inhibitor of DNA binding-3) was shown to be an upstream regulator to protects against the formation of atherosclerosis and the SNP in the coding region of the ID3 gene were directly associated with coronary artery pathology [8]. Such findings matched our results that LFNG and ID3 were lowly expressed in patients with coronary heart diseases, whose low expression levels might contribute to the disease progression. ARG1 regulated the C-reactive protein (CRP) levels whose high levels were risk factors of coronary heart disease [9]. The mutations on PLA2G7 gene have been reported to be associated with coronary artery disease since 15 years ago and some alleles had been proved to increase the risk of coronary heart disease [10]. Again, these findings supported our results that these genes were highly expressed in patients. Other five genes were also closely related with coronary heart disease progression or prognosis in many studies [11-13].

\section{Neutrophil degranulation and coronary heart disease}

We identified 24 pathways related to coronary artery disease. Notably, most of these pathways were associated with immune system. It has been reported that innate and adaptive immune responses played critical roles in the development and progression of coronary heart disease [14]. The primary cause of coronary artery disease, atherosclerosis was widely accepted as a chronic inflammatory disease. It supported our findings.

Neutrophil degranulation was the top one pathway associated with coronary heart disease in our list, which was served as a case study. Neutrophils are important inflammatory cells and bone from marrow-derived white blood cells. Then they migrate from the bloodstream to sites of tissue inflammation and induce inflammation by undergoing burst and degranulation which was illustrated in Figure 4A [15]. The immune function of neutrophils determined the degranulation will alleviate the coronary heart disease. For example, it was noticed that neutrophils degranulation was able to mediate the damage of the vascular and myocardial [16]. Moreover, the function of stimulated neutrophils secreted proteolytic neutral proteases which in further promoted the detachment of endothelial cells from vessel walls and the adherence of platelets to subendothelial collagen and fibronectin [17]. Ricevuti, Mazzone [18] found that neutrophil aggregation and oxygen metabolites release increased in the coronary sinuses of patients with coronary heart disease. Although there were a lot of studies about the
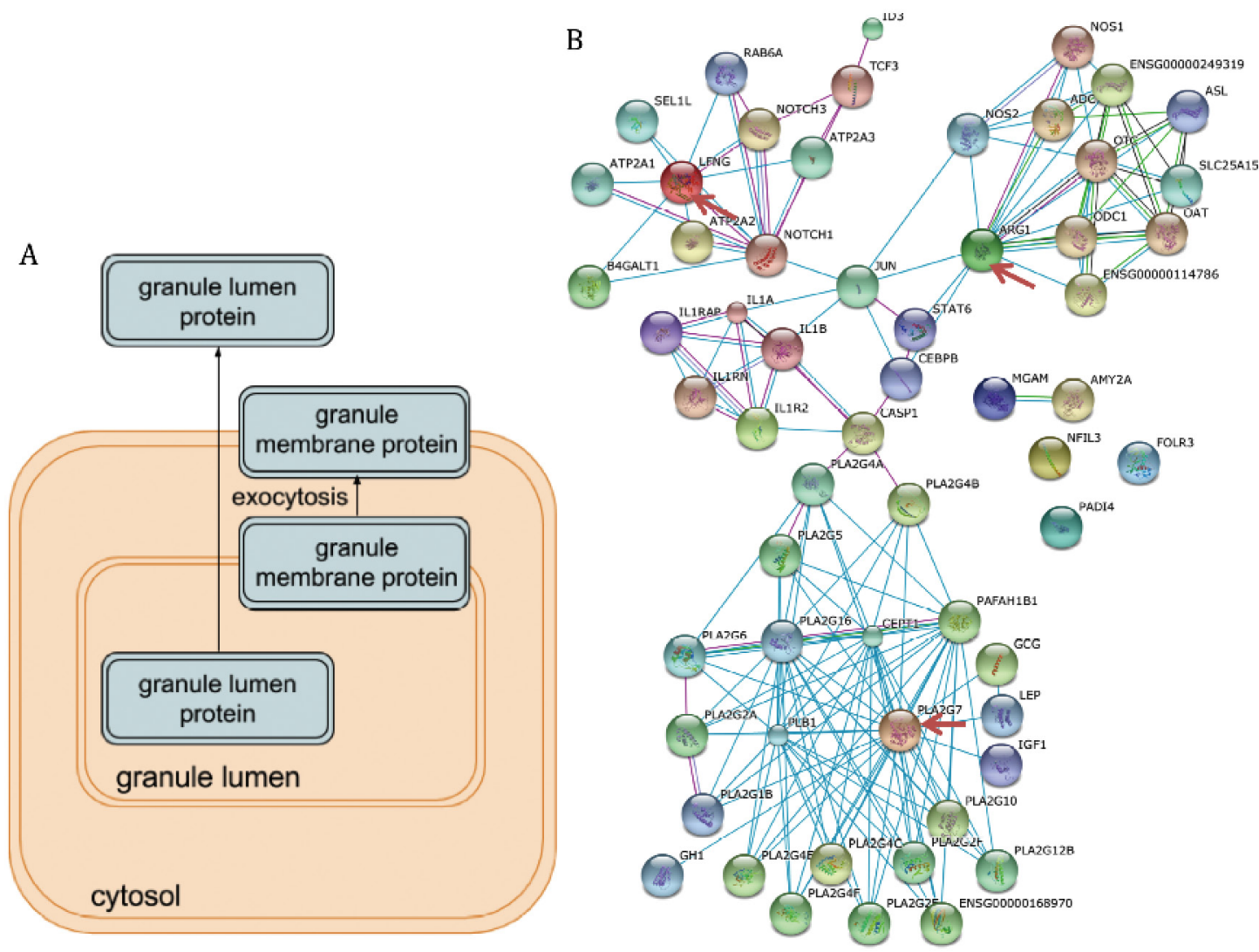

Figure 4: The function analysis of the differentially expressed genes. (A) The pathway illustration of neutrophil degranulation which was the top enriched pathway. (B) The protein-protein interactions between the nine differentially expressed genes and their interactors. The important hubs (LFNG, ARG1, and PLA2G7) were annotated by the red arrows. 
association between neutrophils and coronary heart disease, the attention decreased dramatically in the recent 10 years. We proposed that neutrophils may function critically in the progression of coronary heart disease and serve as the treatment targets.

In conclusion, we aggregated five coronary heart disease microarray datasets from GEO series by systematic analysis. Nine genes which were significantly differentially expressed in all the five datasets were identified. These genes played important roles in patients with coronary artery disease according to the expression levels, protein-protein interactions and enriched pathways. We also concluded that pathways related to immune responses were enriched with the differentially expressed genes and neutrophil degranulation was one of the most important processes.

\section{MATERIALS AND METHODS}

\section{Datasets}

Coronary heart disease and myocardial infarction were used as the keyword to search in GEO series (https:// www.ncbi.nlm.nih.gov/geo/browse/?view=series). After removing datasets which were not obtained from patients' tissues or were short of proper controls, 5 datasets were used to study the expression profiles in patients with coronary heart diseases. The processed data were downloaded as the series matrix using $\mathrm{R}$ package GEOquery [19]. The mRNA expression levels of targeted patients and controls were extracted from all the samples and were transformed into $\log 2$ scale before further analysis. In GSE20681, 99 cases and 99 controls were analyzed by expression profiling microarray using platform GPL4133 [20]. In GSE20680, 52 control and 87 cases were analyzed using the same platform, after intermediates cases were removed [21]. Datasets GSE29532 contained totally 55 samples at different time points, and only the expression profiles on patient admission were extracted in this study, which contained only 6 controls and 8 cases using platform GPL5175 [22]. GSE4128 analyzed 11 controls and 13 patients in Asian Indians using platform GPL13607. In GSE48060, 21 control and 31 myocardial infarction patient groups were analyzed under platform GPL570 [23].

\section{Data pre-processing}

The expression levels of all datasets were transformed into $\log 2$ scale. $\mathrm{R}$ package MetaQC were utilized to finish the preprocessing of datasets [24]. The largest interquartile range (IQR) of expression values were used to represent the genes with multiple probe IDs. The expression levels of 5 datasets were first merged together according to the gene symbol and the genes that appeared in less than 4 datasets were filtered out. Given the fact that most genes were not expressed or not informative in vivo, $20 \%$ of unexpressed genes and $20 \%$ of uninformative genes were removed in order to decrease false positive.

\section{Quality control}

Quantitative quality control measures were calculated to represent the quality of these datasets with the help of MetaQC [24]. The measures included internal quality control index (IQC), external quality control index (EQC), accuracy quality control index for genes or pathways (AQCg and AQCp) and consistency of differential expression quality control (CQCg and CQCp) indexes. IQC represented the internal homogeneity of coexpression, which identified potentially inconsistent or outlier studies from quantified co-expression dissimilarity. EQC index was calculated with the supervision of external pathway database MSigDB. The Biocarta and all pathways of version 5.2 from MSigDB were applied to evaluate its consistency with each study. AQC and CQC aimed at quantifying the reproducibility of differentially expressed genes or pathways detected in an individual study compared to those detected by systematic analysis from all other studies.

Principal component analysis (PCA) biplots were plotted with the help of MetaQC to visualize the quality of studies in systematic analysis. The six quality control measures were projected into a $2 \mathrm{D}$ space; that is, the coordinates of each quality criterion were determined by its correlation to the first two principal coordinates.

\section{Identification of differentially expressed genes}

MetaDE package provides functions for conducting 5 different systematic analysis methods for differential expression analysis [25]; that is, Fisher, adaptively weighted Fisher (AW), minimum p-value (minP), maximum $\mathrm{p}$-value (maxP) and $\mathrm{r}^{\text {th }}$-ordered $\mathrm{p}$-value (roP). Moderated t-test was used to decide the differentially expressed genes for a single dataset. The heatmap of the differentially expressed genes under 0.1 FDR threshold across studies where created. To assess the performance of these different methods, we compared the numbers of detected differentially expressed genes from different methods under different $\mathrm{p}$-value thresholds using detection competency curves.

\section{Function analysis}

The pathway enrichment was carried out using Reactome database and FDR adjustment was applied to identify significantly enriched pathways [26, 27]. The pathways shared by at least three datasets were plotted as heatmap. Protein-protein interactions of the identified differentially expressed genes were also analyzed using STRING v10.0 online tool that visualizes known and predicted protein-protein interactions [28]. 


\section{CONFLICTS OF INTEREST}

The authors declare no conflicts of interest.

\section{REFERENCES}

1. Mozaffarian D, Benjamin EJ, Go AS, Arnett DK, Blaha MJ, Cushman M, de Ferranti S, Després JP, Fullerton HJ, Howard VJ, Huffman MD, Judd SE, Kissela BM, et al, and American Heart Association Statistics Committee and Stroke Statistics Subcommittee. Heart disease and stroke statistics - 2015 update: a report from the American Heart Association. Circulation. 2015; 131:e29-322.

2. Ford ES, Ajani UA, Croft JB, Critchley JA, Labarthe DR, Kottke TE, Giles WH, Capewell S. Explaining the decrease in U.S. deaths from coronary disease, 1980-2000. N Engl J Med. 2007; 356:2388-98.

3. Sinnaeve PR, Donahue MP, Grass P, Seo D, Vonderscher J, Chibout SD, Kraus WE, Sketch M Jr, Nelson C, Ginsburg GS, Goldschmidt-Clermont PJ, Granger CB. Gene expression patterns in peripheral blood correlate with the extent of coronary artery disease. PLoS One. 2009; 4:e7037.

4. Ein-Dor L, Kela I, Getz G, Givol D, Domany E. Outcome signature genes in breast cancer: is there a unique set? Bioinformatics. 2005; 21:171-78.

5. Ntzani EE, Ioannidis JP. Predictive ability of DNA microarrays for cancer outcomes and correlates: an empirical assessment. Lancet. 2003; 362:1439-44.

6. Barrett T, Wilhite SE, Ledoux P, Evangelista C, Kim IF, Tomashevsky M, Marshall KA, Phillippy KH, Sherman PM, Holko M, Yefanov A, Lee H, Zhang N, et al. NCBI GEO: archive for functional genomics data sets - update. Nucleic Acids Res. 2013; 41:D991-95.

7. Rusanescu G, Weissleder R, Aikawa E. Notch signaling in cardiovascular disease and calcification. Curr Cardiol Rev. 2008; 4:148-56.

8. Manichaikul A, Rich SS, Perry H, Yeboah J, Law M, Davis M, Parker M, Ragosta M, Connelly JJ, McNamara CA, Taylor AM. A functionally significant polymorphism in ID3 is associated with human coronary pathology. PLoS One. 2014; 9:e90222.

9. Vinayagamoorthy $\mathrm{N}, \mathrm{Hu} \mathrm{HJ}$, Yim $\mathrm{SH}$, Jung $\mathrm{SH}$, Jo J, Jee SH, Chung YJ. New variants including ARG1 polymorphisms associated with C-reactive protein levels identified by genome-wide association and pathway analysis. PLoS One. 2014; 9:e95866.

10. Wang Q, Hao Y, Mo X, Wang L, Lu X, Huang J, Cao J, Li H, Gu D. PLA2G7 gene polymorphisms and coronary heart disease risk: a meta-analysis. Thromb Res. 2010; 126:498-503.

11. Sivapalaratnam S, Basart H, Watkins NA, Maiwald S, Rendon A, Krishnan U, Sondermeijer BM, Creemers EE, Pinto-Sietsma SJ, Hovingh K, Ouwehand WH, Kastelein JJ, Goodall AH, Trip MD. Monocyte gene expression signature of patients with early onset coronary artery disease. PLoS One. 2012; 7:e32166.

12. Eyre S, Bowes J, Diogo D, Lee A, Barton A, Martin P, Zhernakova A, Stahl E, Viatte S, McAllister K, Amos CI, Padyukov L, Toes RE, et al, and Biologics in Rheumatoid Arthritis Genetics and Genomics Study Syndicate, and Wellcome Trust Case Control Consortium. High-density genetic mapping identifies new susceptibility loci for rheumatoid arthritis. Nat Genet. 2012; 44:1336-40.

13. Chen X, Chen X, Xu Y, Yang W, Wu N, Ye H, Yang JY, Hong Q, Xin Y, Yang MQ, Deng Y, Duan S. Association of six CpG-SNPs in the inflammation-related genes with coronary heart disease. Hum Genomics. 2016; 10:21.

14. Fernández-Ruiz I. Immune system and cardiovascular disease. Nat Rev Cardiol. 2016; 13:503.

15. Lacy P. Mechanisms of degranulation in neutrophils. Allergy Asthma Clin Immunol. 2006; 2:98-108.

16. Lagrand WK, Visser CA, Hermens WT, Niessen HW, Verheugt FW, Wolbink GJ, Hack CE. C-reactive protein as a cardiovascular risk factor: more than an epiphenomenon? Circulation. 1999; 100:96-102.

17. Madjid M, Awan I, Willerson JT, Casscells SW. Leukocyte count and coronary heart disease: implications for risk assessment. J Am Coll Cardiol. 2004; 44:1945-56.

18. Ricevuti G, Mazzone A, De Servi S, Specchia G, Fratino P. New trends in coronary artery disease: the role of granulocyte activation. Atherosclerosis. 1989; 78:261-65.

19. Davis S, Meltzer PS. GEOquery: a bridge between the Gene Expression Omnibus (GEO) and BioConductor. Bioinformatics. 2007; 23:1846-47.

20. Beineke P, Fitch K, Tao H, Elashoff MR, Rosenberg S, Kraus WE, Wingrove JA, Investigators $\mathrm{P}$, and PREDICT Investigators. A whole blood gene expression-based signature for smoking status. BMC Med Genomics. 2012; 5:58.

21. Elashoff MR, Wingrove JA, Beineke P, Daniels SE, Tingley WG, Rosenberg S, Voros S, Kraus WE, Ginsburg GS, Schwartz RS, Ellis SG, Tahirkheli N, Waksman R, et al. Development of a blood-based gene expression algorithm for assessment of obstructive coronary artery disease in non-diabetic patients. BMC Med Genomics. 2011; 4:26.

22. Silbiger VN, Luchessi AD, Hirata RD, Lima-Neto LG, Cavichioli D, Carracedo A, Brión M, Dopazo J, García-García F, dos Santos ES, Ramos RF, Sampaio MF, Armaganijan D, et al. Novel genes detected by transcriptional profiling from whole-blood cells in patients with early onset of acute coronary syndrome. Clin Chim Acta. 2013; 421:184-90.

23. Suresh R, Li X, Chiriac A, Goel K, Terzic A, Perez-Terzic C, Nelson TJ. Transcriptome from circulating cells suggests dysregulated pathways associated with long-term recurrent events following first-time myocardial infarction. J Mol Cell Cardiol. 2014; 74:13-21.

24. Kang DD, Sibille E, Kaminski N, Tseng GC. MetaQC: objective quality control and inclusion/exclusion criteria for genomic meta-analysis. Nucleic Acids Res. 2012; 40:e15. 
25. Wang X, Lin Y, Song C, Sibille E, Tseng GC. Detecting disease-associated genes with confounding variable adjustment and the impact on genomic meta-analysis: with application to major depressive disorder. BMC Bioinformatics. 2012; 13:52.

26. Haw R, Hermjakob H, D'Eustachio P, Stein L. Reactome pathway analysis to enrich biological discovery in proteomics data sets. Proteomics. 2011; 11:3598-613.

27. Fabregat A, Sidiropoulos K, Garapati P, Gillespie M, Hausmann K, Haw R, Jassal B, Jupe S, Korninger F,
McKay S, Matthews L, May B, Milacic M, et al. The Reactome pathway Knowledgebase. Nucleic Acids Res. 2016; 44:D481-87.

28. Szklarczyk D, Franceschini A, Wyder S, Forslund K, Heller D, Huerta-Cepas J, Simonovic M, Roth A, Santos A, Tsafou KP, Kuhn M, Bork P, Jensen LJ, von Mering C. STRING v10: protein-protein interaction networks, integrated over the tree of life. Nucleic Acids Res. 2015; 43:D447-52. 